\title{
Synchrotron Mössbauer Reflectometry - Recent Applications in Multilayer Magnetism
}

\author{
D.L. Nagy, L. Bottyán, L. DeÁk and M. Major
}

KFKI Research Institute for Particle and Nuclear Physics

P.O.B. 49, 1525 Budapest, Hungary

\begin{abstract}
Grazing incidence nuclear resonant scattering of synchrotron radiation (synchrotron Mössbauer reflectometry) has recently been efficiently applied to study the magnetic structure of multilayers. Principles and recent experiments of synchrotron Mössbauer reflectometry are briefly reviewed. The orientation of the layer magnetization in an antiferromagnetically coupled multilayer is determined both from time-integral and time-differential specular synchrotron Mössbauer reflectometry experiments. Spin-flop phenomena are studied. Off-specular (diffuse) scattering gives information on the in-plane antiferromagnetic domain structure.
\end{abstract}

PACS numbers: 75.70.-i, 75.25.+z, 75.30.Gw, 76.80.+y

\section{Introduction}

Total external reflection (TER) of X-rays [1] and neutrons [2] from flat surfaces are phenomena dating back to the first half of the twentieth century. The real part of the index of refraction $n$ of most materials for thermal neutrons and of all materials for non-resonant $\mathrm{X}$-rays is by about $10^{-5}$ less than unity. At low enough angles of grazing incidence $\Theta<\Theta_{c}=\sqrt{2(1-n)}$ the waves are totally reflected. The intensity of the reflected specular beam for $\Theta<\Theta_{c}$ rapidly decreases with increasing wave vector transfer $Q=2 k \sin \Theta$, where $k$ is the length of the wave vector of the incident radiation. In a stratified medium, reflected and refracted beams appear at each interface. The interference of the reflected beams leads to patterns of the reflectivity vs. wave vector transfer spectrum $R(Q)$ that bear information on the depth profile of the index of refraction $n(z)$, the argument $z$ being the co-ordinate perpendicular to the sample surface. $R(Q)$ can be calculated from $n(z)$, e.g. using the method of characteristic matrices [3]. Therefore, in frames of a 
given model for the stratified system, $n(z)$ can be reconstructed (the parameters of the model can be fitted) from $R(Q)=|r(Q)|^{2}$, where $r(Q)$ is the reflectivity amplitude. This latter approach is the basic idea of specular X-ray and neutron reflectometry, two methods that can be used for mapping the electron density and the isotopic/magnetic structure of thin films, respectively. In fact, the coherent forward scattering of a scalar wave of momentum much higher than that of the scatterers can be described [4] by the index of refraction close to unity

$$
n=1+\frac{2 \pi N}{k^{2}} f,
$$

where $N$ is the density of scatterers and $f$ is the scattering amplitude. The electron density for non-resonant X-rays or nuclear and magnetic scattering length density for neutrons is implied in the latter quantity.

X-ray reflectometry may optionally be performed with nuclear resonant photons. We shall call this technique, henceforth, Mössbauer reflectometry (MR). MR benefits from the fact that, close to the nuclear resonance, the photon scattering amplitude $f$ is strongly energy-dependent and contains the matrix elements of the hyperfine interactions. MR is therefore suitable to study the magnetic structure of thin films.

A serious limitation of MR with conventional sources [5] is the small $\left(\sim 10^{-5}\right)$ solid angle involved. Due to its high collimation, synchrotron radiation (SR) is much better suited for reflectometric experiments than radioactive sources. Synchrotron Mössbauer reflectometry (SMR) is the application of grazing incidence nuclear resonant scattering of SR [6] to thin film and multilayer structure analysis. SMR has recently been reviewed in various papers [7-10]. SMR and polarised neutron reflectometry can be mapped onto each other and a common optical formalism exists [11].

An important step towards the realization of SMR was the observation [12] of the total reflection peak $[12,13]$, i.e. the high number of delayed photons appearing close to the critical angle of the electronic TER. This peak is formally identified with the zeroth-order Bragg peak in nuclear resonant specular reflection. The last half decade saw an increasing number of SMR experiments since the pioneering work by Toellner et al. [14], including a recent extension of the technique to off-specular (diffuse) scattering [15]. In this paper, as an update of a previous article [10] we shall briefly review the principles and some methodological aspects of the application of SMR to magnetic structure analysis of thin films and multilayers. It will be shown that it is possible to study the orientation of the layer magnetization, spin-flop phenomena and magnetic domain structure. Since the general aspects of the application of nuclear resonant scattering of SR are described by a paper of Leupold in the present volume [16], we will refer to it whenever possible. Throughout the present paper the $14.413 \mathrm{keV}$ transition of ${ }^{57} \mathrm{Fe}$ is used. 


\section{Specular and off-specular SMR}

A sketch of the experimental arrangement of an SMR experiment is shown in Fig. 1. The photons from the high-resolution monochromator hit the sample mounted on a two-circle goniometer of adjustable height at an angle of grazing incidence $\omega$. Further slits and optional focusing elements (not shown in the figure) are installed both upstream and downstream the monochromator (cf. Fig. 4 of Ref. [16]). The reflected (scattered) photons are detected by an avalanche photo diode (APD), the aperture of which may be limited by a slit in front of the detector. The adjustable detector height defines the scattering angle $2 \Theta$.

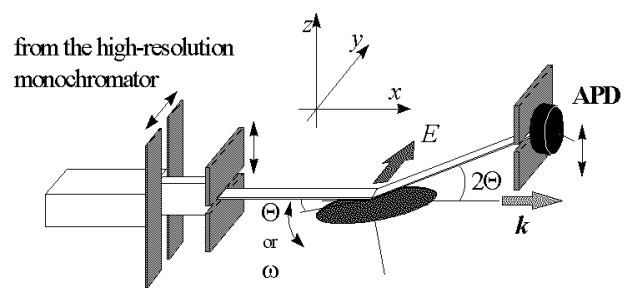

Fig. 1. Experimental setup of an SMR experiment.

An SMR measurement is performed in either time integral or time differential regime. Time integral SMR (TISMR) records the total number of delayed photons from $t_{1}$ to $t_{2}$ as a function of $\omega$ and/or $\Theta$ (for details see below). Here $t_{1}$ is a few nanoseconds determined by the bunch quality of the radiation source and by the dead time of the detector and the electronics, while $t_{2}$ is set to a value somewhat below the bunch repetition time of the storage ring. Time differential (TD) SMR is a time response measurement performed at various fixed values of $\omega$ and $\Theta$. Like in the forward scattering case, hyperfine interaction results in quantum beats of the time response [16].

Position-sensitive detectors have recently been increasingly applied in X-ray and neutron reflectometry in order to map the scattered intensity in a single experiment on the whole $(\omega, \Theta)$ plane. Although APD arrays are being tested at various synchrotron facilities, as yet, TISMR experiments have only been performed in two different single-parameter geometries, viz., $\Theta-2 \Theta$ scan and $\omega$ scan. In a $\Theta-2 \Theta$ scan the sample orientation and the detector height are simultaneously changed fulfilling the constraint of specular reflection, $\omega=\Theta$. In an $\omega$-scan experiment $2 \Theta$ is fixed and $\omega$ is varied. As a rule, a scan of the prompt photons (i.e., X-ray reflectometry) is recorded along with a delayed TISMR scan.

In a $\Theta-2 \Theta$ experiment the wave vector transfer $Q$ is perpendicular to the sample surface. For a periodic multilayer, in the first Born approximation (kinematic theory), Bragg maxima appear at $Q=\sqrt{(2 \pi / d)^{2}+Q_{c}^{2}}$, where $d$ is the structural or hyperfine (magnetic) period length perpendicular to the film plane 
and $Q_{c}$ is the critical wave vector transfer of the TER (typically about $0.5 \mathrm{~nm}^{-1}$ ). Thus a $\Theta-2 \Theta$ scan reveals the plane-perpendicular structure with the provision that the film is homogeneous in its plane. Should this not be the case, the intensity of the specular reflection is reduced. Lateral dimensions of inhomogeneities such as structural and magnetic roughness, waviness, magnetic domains, etc., however, cannot be further studied in a $\Theta-2 \Theta$ experiment.

In an $\omega$-scan experiment the condition of specular reflection is not fulfilled for $\omega \neq \Theta$. Off-specular scattered intensity is only significant in case of lateral inhomogeneities. In fact, for the small values of $\omega$ and $\Theta$ in this experiment, the perpendicular-to-plane component of the wave vector transfer is constant $(Q z=2 k \Theta)$ while varying $\omega$, the in-plane parallel-to-beam (longitudinal) component of the wave vector transfer is scanned: $Q_{x}=2 k \Theta(\omega-\Theta)$. In order to have significant intensity, the detector height is set to meet the $Q_{z}$ value of a Bragg peak. In the first Born approximation the width of the $\omega$ scan (i.e., $Q_{x}$ scan) is inversely proportional to the longitudinal correlation length $\xi$

$$
\xi=\frac{2 \pi}{\Delta Q_{x}}=\frac{\pi}{k \Theta \Delta \omega},
$$

where $\Delta Q_{x}$ and $\Delta \omega$ are the peak widths of the $Q_{x}$ and $\omega$ scans, respectively. $\xi$ is the correlation length of the quantity of the perpendicular-to-plane periodicity of which the Bragg peak is due to. Therefore, setting $2 \Theta$ in an $\omega$-scan experiment to an electronically forbidden pure nuclear reflection [16] the lateral correlation length of inhomogeneities of the hyperfine interaction (magnetic roughness, magnetic domains) can be determined.

In contrast to TISMR, TDSMR experiments have only been performed in specular reflection so far.

\section{Layer magnetization direction in coupled multilayers}

The direction of the layer magnetization is an important issue in thin film magnetism. Its dependence on the applied magnetic field and temperature yields information on interlayer coupling and magnetic anisotropy. Both TISMR and TDSMR can be used to determine the layer magnetization direction in thin films and multilayers.

The origin of pure nuclear superstructure reflections of AF origin in TISMR has been explained in Ref. [16] (Sec. 4 and Figs. 5 and 6). For directions of the layer magnetization between beam-parallel and beam-perpendicular orientations in an AF-coupled multilayer, the relative intensity of the AF peak is reduced, giving, thereby, an excellent tool for studying the process of magnetic saturation. TISMR was applied to follow the saturation process in Zerodur $/\left[{ }^{57} \mathrm{Fe}(2.55 \mathrm{~nm}) /\right.$ $\left.{ }^{\text {nat }} \mathrm{FeSi}(1.5 \mathrm{~nm})\right]_{10}$ multilayer [17] and in $\mathrm{MgO}(001) /\left[{ }^{57} \mathrm{Fe}(1.43 \mathrm{~nm}) / \mathrm{Cr}(3.06 \mathrm{~nm})\right]_{16}$ superlattice [10]. However, since the AF peak is suppressed for any beam-perpendicular direction of the layer magnetization, a TISMR experiment cannot distin- 
guish between out-of-plane and in-plane beam-perpendicular components of the layer magnetization.

Due to the linear polarization of SR, the quantum-beat patterns measured in a time-differential nuclear resonant experiment contain more detailed (but not full, see below) 3D information on the direction of the hyperfine magnetic field (i.e., of the magnetization). This has been explained in Ref. [16] (Sec. 2 and Fig. 1). Out-of-plane magnetization was found by TDSMR in Fe/Ag superlattices [18] and in the inner region of 7 monolayers fcc Fe sandwiched between ferromagnetic fcc Co layers stabilised by $\mathrm{Cu}[19]$.
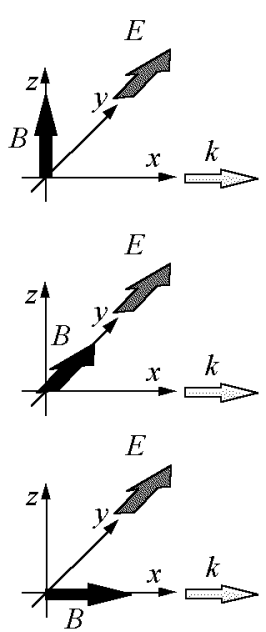

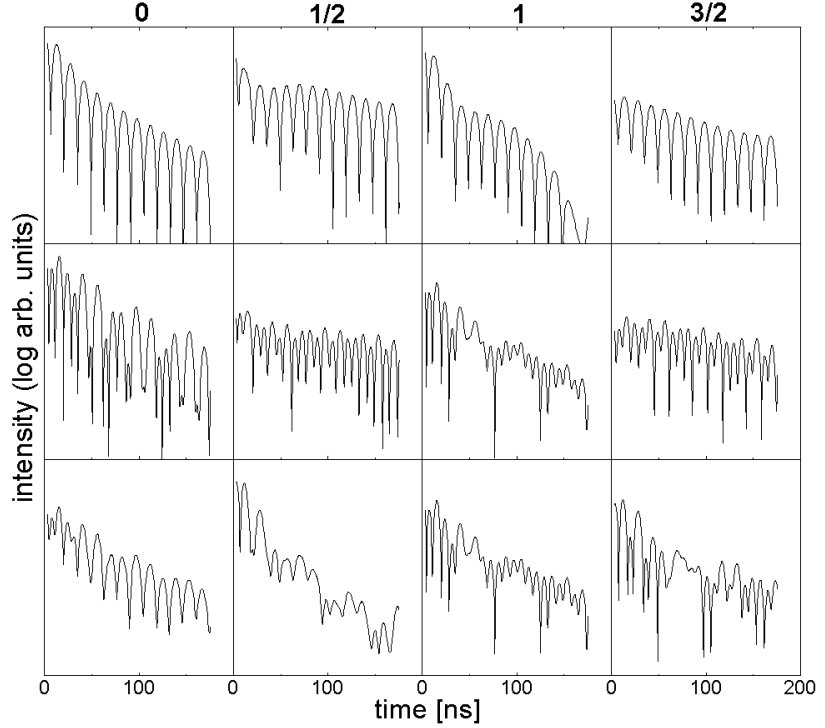

Fig. 2. Calculated time response curves of an AF-coupled $\left[{ }^{57} \mathrm{Fe}(2.00 \mathrm{~nm}) /\right.$ $\mathrm{Cr}(2.62 \mathrm{~nm})]_{20}$ multilayer for three different directions of the hyperfine field $\boldsymbol{B}$ (the hyperfine field of one of the two sublattices is not shown). The scattering plane is perpendicular to the electric field vector $\boldsymbol{E}$ of the SR. Integer and half-integer numbers indicate the order of the structural and hyperfine (magnetic) Bragg reflections, respectively.

Figure 2 shows calculated TDSMR spectra of an AF-coupled $\left[{ }^{57} \mathrm{Fe}(2.00 \mathrm{~nm}) /\right.$ $\mathrm{Cr}(2.62 \mathrm{~nm})]_{20}$ multilayer for three different directions of the hyperfine field $\boldsymbol{B}$ and at four different values of $\Theta$ corresponding to the $0-, 1 / 2-, 1$ - and $3 / 2$-order Bragg reflections. The 0 -order reflection is the total reflection peak, the 1 -order reflection is the structural Bragg peak, while the half-order reflections are pure nuclear superstructure peaks of AF origin. The quantum-beat patterns at the structural Bragg peak belonging to magnetization directions parallel to the beam or parallel to the polarization of SR are identical. This feature has been mentioned in Ref. [16] (Sec. 2 and Fig. 2) for the forward scattering case and can be easily understood by observing the stick diagrams in Fig. 1 of Ref. [16]. Indeed, for a uniaxial antiferro- 
magnet, if the hyperfine magnetic field is parallel to the beam for one sublattice, it is antiparallel for the other. Therefore, left and right circular polarised transitions appear at the same energy for the one and the other sublattice, respectively. Consequently, all transitions can interfere with each other, which results in the same quantum-beat pattern as if the hyperfine field is parallel to the polarization of the SR. In this latter case all transition remain $\sigma$-polarised for a uniaxial antiferromagnet so that no change is expected as compared to the case of a ferromagnet shown in the left-hand side of Fig. 2 of Ref. [16]. For a uniaxial antiferromagnet not only the quantum-beat patterns for in-plane magnetization parallel and perpendicular to the beam but for any in-plane direction of the magnetization are identical.

In view of this, it is surprising that, as seen in Fig. 2, the same quantum-beat patterns are markedly different for the 0-order (total reflection) peak. The reason for this fact is the limited penetration depth of SR in the total reflection region. Accordingly, the uppermost Fe layer contributes much more to the TDSMR quantum-beat pattern than the second, etc. Therefore, the argument of the previous paragraph is no longer valid and the time response curves resemble more to the left column of Fig. 2 of Ref. [16]. In contrast to forward scattering, TDSMR spectra contain information on the orientation of layer magnetization of a uniaxial antiferromagnet.

TDSMR spectra of Zerodur $/\left[{ }^{57} \mathrm{Fe}(2.55 \mathrm{~nm}) /{ }^{\mathrm{nat}} \mathrm{FeSi}(1.5 \mathrm{~nm})\right]_{10}$ measured in magnetic field much below saturation were indicative of a $62^{\circ}$ misalignment of the sublattice magnetizations with respect to the perpendicular-to-the-field alignment. This phenomenon was attributed to a depth-dependent bilinear coupling $[17,20]$. Having revisited the problem it was shown that finiteness of the multilayer stacking [21] alone leads to a global twist of the sublattice magnetizations in small external fields so that an upper uncompensated finite AF-coupled block and a single uncoupled FM layer on the substrate can even better describe the measured time response curves [22].

\section{Spin-flop phenomena}

An interesting model system in multilayer magnetism is a periodic AF-coupled $\mathrm{Fe} / \mathrm{Cr}$ superlattice with even number of Fe layers. When the external magnetic field is aligned along the easy axis of the Fe layers parallel/antiparallel to the layer magnetizations, the anisotropy-stabilised configuration becomes energetically unfavourable at a certain critical in-plane field strength and a sudden magnetization reorientation is expected in a finite multilayer stack [21-23] with surface spin-flop $[24,25]$ or bulk spin-flop (BSF) [26] scenarios, in cases of uniaxial and fourfold in-plane anisotropy, respectively. These processes are associated with major jumps of the direction of one or more layer magnetization, but, as a rule, only with minute changes of the net magnetization. It is, therefore, not easy to identify a spin-flop transition with methods of classical magnetism. 
As shown in the previous section, SMR is a sensitive tool to determine the layer magnetization directions in thin films and multilayers. Therefore, SMR is especially suitable for studying spin-flop phenomena. We have recently reported on TISMR of the (bulk) spin-flop in an AF-coupled Fe/Cr superlattice with a fourfold in-plane anisotropy [27, 28].

The experiment at the nuclear resonance beamline ID18 of the European Synchrotron Radiation Facility was performed on a $\mathrm{MgO}(001) /\left[{ }^{57} \mathrm{Fe}(26 \AA) /\right.$ $\mathrm{Cr}(13 \AA)]_{20}$ multilayer [28]. The epitaxial relationship on $\mathrm{MgO}(001)$ substrate is $\mathrm{MgO}(001)[110] / \mathrm{Fe}(001)[100]$, therefore the magnetization of the individual $\mathrm{Fe}$ layers points parallel or anti-parallel to either of the Fe[010] or Fe[100] axes in the film plane. The initial magnetic state of the multilayer was carefully prepared by applying external magnetic field as described below. The scattering plane was vertical and the wave vector $k$ of the incoming resonant beam was perpendicular to the in-plane magnetic field, as shown in Fig. 1. First the sample was saturated in-plane in one of the easy directions then the external field was decreased to zero. At this stage the sample was turned in-plane by $90 \mathrm{deg}$ and TISMR scans were recorded as a function of increasing external magnetic fields. Figure 3 shows the

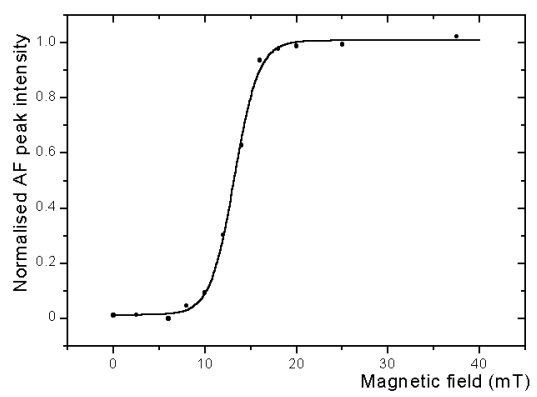

Fig. 3. Normalised intensity of the $\mathrm{AF}$ peak of a $\mathrm{MgO}(001) /\left[{ }^{57} \mathrm{Fe}(26 \AA) / \mathrm{Cr}(13 \AA)\right]_{20}$ multilayer during bulk spin flop. The continuous line is to guide the eyes.

normalised intensity of the AF peak as a function of the increasing magnetic field. The appearance of the 1/2- and 3/2-order AF reflections around $13.2 \mathrm{mT}$ is a direct evidence of the $90-\mathrm{deg}$ rotation of the Fe layer magnetizations, i.e. of the bulk-spin-flop process. Due to the fourfold symmetry of the anisotropy, this state is preserved after the magnetic field is removed. A detailed analysis of the TISMR scans and the corresponding TDSMR spectra will be published elsewhere.

\section{Antiferromagnetic domains in multilayers}

Domain structure of AF-coupled multilayers is an issue of both theoretical and technological importance. Domain-size-dependent resistance noise, for example, may be as large as to limit GMR-sensor applications [29]. It is extremely 
difficult to visualise in-plane AF domains in a multilayer of a few nm thickness. In fact, Kerr-microscopy has been performed so far only on thick trilayers [30, 31].

Therefore indirect methods like resistance noise [29] and magnetoresistance [32] measurements, off-specular non-polarised [33] and polarised neutron reflectometry $[34,35]$ and, recently, soft-X-ray resonant magnetic diffuse scattering [36] have been used to estimate the AF-domain-size distribution in magnetic multilayers.

Off-specular SMR is, as shown in Sec. 2, also suitable to investigate the in-plane correlation length of AF domains in coupled multilayers. Figure 4 shows off-specular SMR scans of a $\mathrm{MgO}(001) /\left[{ }^{57} \mathrm{Fe}(26 \AA) / \mathrm{Cr}(13 \AA)\right]_{20}$ multilayer at the AF reflection of $\Theta=0.4 \mathrm{deg}$ in two different states, depending on the magnetic prehistory [15]. The domain size or, more precisely, the correlation length can be evaluated from the width of the off-specular $\omega$-scan using Eq. (2). The broad line in scan (a) corresponds to AF microdomains of correlation length $\xi \approx 2.6 \mu \mathrm{m}$. In contrast to this, scan (b) is the sum of a broad diffuse shoulder ( $22 \%$ of the total area) and a narrow specular line ( $78 \%)$. In this state $22 \%$ of the multilayer consists of microdomains $(\xi \approx 2.6 \mu \mathrm{m})$ while the majority of the multilayer contains large domains. Due to the finite aperture of the detector, only a lower limit of the correlation length $(\xi>16.5 \mu \mathrm{m})$ can be deduced from the width of the specular peak.

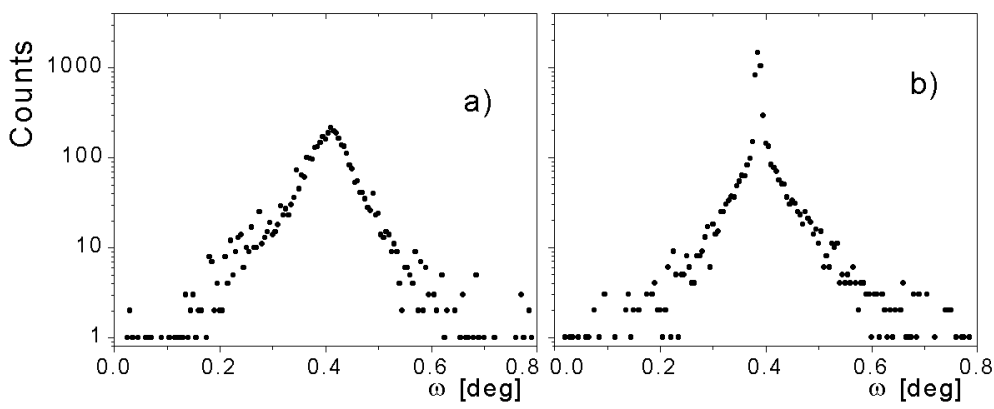

Fig. 4. Off-specular SMR scans of a $\mathrm{MgO}(001) /\left[{ }^{57} \mathrm{Fe}(26 \AA) / \mathrm{Cr}(13 \AA)\right]_{20}$ multilayer at the AF reflection: (a) small domain state, (b) large domain state.

The typical data collection time of an off-specular SMR scan is about $30 \mathrm{~min}$, i.e., by one order of magnitude shorter than the typical time necessary for an off-specular polarised neutron reflectometry or off-specular soft-X-ray resonant magnetic diffuse scattering experiment. This holds true, however, only for ${ }^{57} \mathrm{Fe}$-containing AF-coupled multilayers.

\section{Summary}

SMR has become an efficient tool in studying magnetic structure of multilayers. AF-coupling results in pure nuclear superstructure reflections in time integral SMR experiments. The intensity of the AF reflection is a measure of the in-plane orientation of the layer magnetization. Time-differential specular SMR 
experiments can be used to determine both in-plane and out-of-plane layer magnetization components. Bulk-spin-flop transition results in a sudden change of the AF peak intensity. Off-specular (diffuse) SMR scans show broad shoulders for AF microdomains and sharp specular lines for large domains (a few tens of $\mu \mathrm{m}$ or more).

\section{Acknowledgments}

The authors acknowledge the European Synchrotron Radiation Facility for provision of synchrotron radiation facilities. They are very much grateful to Drs. O. Leupold and R. Rüffer (ESR), the members of IKS of the University of KU Leuven, the nuclear resonance groups of the ESRF and the University of Hamburg for many stimulating discussions. Support by the project No. T 029409 of the Hungarian Scientific Research Fund (OTKA) and by the Hungarian Academy of Sciences, Contract No. 2000-103 2,3 is gratefully acknowledged.

\section{References}

[1] H. Kiessig, Ann. Phys. (Leipzig) 10, 715 (1931).

[2] E. Fermi, W. Zinn, Phys. Rev. 70, 103 (1946).

[3] M. Born, E. Wolf, Principles of Optics, Pergamon Press, Oxford 1970, p. 51.

[4] M. Lax, Rev. Mod. Phys. 23, 287 (1951).

[5] S.M. Irkaev, M.A. Andreeva, V.G. Semenov, G.N. Belozerskii, O.V. Grishin, Nucl. Instrum. Methods B 74, 545 (1993).

[6] E. Gerdau, R. Rüffer, H. Winkler, W. Tolksdorf, C.P. Klages, J.P. Hannon, Phys. Rev. Lett. 54, 835 (1985).

[7] D.L. Nagy, L. Bottyán, L. Deák, J. Dekoster, G. Langouche, V.G. Semenov, H. Spiering, E. Szilágyi, in: Mössbauer Spectroscopy in Materials Science, Eds. M. Miglierini, D. Petridis, Kluwer Academic Publishers, 1999, p. 323.

[8] R. Röhlsberger, Hyperfine Interact. 123/124, 301 (1999).

[9] A.I. Chumakov, L. Niesen, D.L. Nagy, E.E. Alp, Hyperfine Interact. 123/124, 427 (1999).

[10] D.L. Nagy, L. Bottyán, L. Deák, E. Szilágyi, H. Spiering, J. Dekoster, G. Langouche, Hyperfine Interact. 126, 353 (2000).

[11] L. Deák, L. Bottyán, D.L. Nagy, H. Spiering, Physica B 297, 113 (2001).

[12] A.Q.R. Baron, J. Arthur, S.L. Ruby, A.I. Chumakov, G.V. Smirnov, G.S. Brown, Phys. Rev. B 50, 10354 (1994).

[13] L. Deák, L. Bottyán, D.L. Nagy, Hyperfine Interact. 92, 1083 (1994).

[14] T.L. Toellner, W. Sturhahn, R. Röhlsberger, E.E. Alp, C.H. Sowers, E.E. Fullerton, Phys. Rev. Lett. 74, 3475 (1995).

[15] D.L. Nagy, L. Bottyán, B. Croonenborghs, L. Deák, B. Degroote, J. Dekoster, H.J. Lauter, V. Lauter-Pasyuk, O. Leupold, M. Major, J. Meersschaut, O. Nikonov, A. Petrenko, R. Rüffer, H. Spiering, E. Szilágyi, submitted to Phys. Rev. Lett.

[16] O. Leupold, Acta Phys. Pol. A 100, (2001). 
[17] L. Bottyán, J. Dekoster, L. Deák, A.Q.R. Baron, S. Degroote, R. Moons, D.L. Nagy, G. Langouche, Hyperfine Interact. 113, 295 (1998).

[18] J. Dekoster, J. Meersschaut, B. Degroote, S. Degroote, C. L’abbé, G. Koops, M.J. Prandolini, T. Phalet, L. Vanneste, H.D. Pfannes, D.L. Nagy, L. Bottyán, R. Rüffer, O. Leupold, G. Langouche, Hyperfine Interact. 126, 349 (2000).

[19] C. Carbone, A. Dallmeyer, M.C. Malagoli, K. Maiti, J. Wingbermühle, W. Eberhartd, D.L. Nagy, Bottyán, L. Deák, E. Szilágyi, L.R. Rüffer, O. Leupold, in: ESRF Highlights 1999, European Synchrotron Radiation Facility, Grenoble 2000, p. 60 .

[20] J. Kohlhepp, M. Valkier, A. van der Graaf, F.J.A. den Broeder, Phys. Rev. B 55, R696 (1997).

[21] F.C. Nötermann, R.L.Stamps, A.S. Carrico, R.E. Camley, Phys. Rev. B 46, 10847 (1992).

[22] M. Major, L. Bottyán, L. Deák, D.L. Nagy, in: Condensed Matter Studies by Nuclear Methods (Proc. XXXII, Zakopane School of Physics, Zakopane), Eds. E.A. Görlich, A. Pedziwiatr, Institute of Physics, Jagellonian University and H. Niewodniczanski Institute of Nuclear Physics, Cracow 1999, p. 165.

[23] A.L. Dantas, A.S. Carriço, Phys. Rev. B 59, 1223 (1999).

[24] R.W. Wang, D.L. Mills, E.E. Fullerton, J.E. Mattson, S.D. Bader, Phys. Rev. Lett. 72, 920 (1994).

[25] N.S. Almeida, D.L. Mills, Phys. Rev. B 52, 13504 (1995).

[26] K. Temst, E. Kunnen, V.V. Moshchalkov, H. Maletta, H. Fritzsche, Y. Bruynseraede, Physica B 276-278, 684 (2000).

[27] L. Bottyán, L. Deák, J. Dekoster, E. Kunnen, G. Langouche, J. Meersschaut, M. Major, D.L. Nagy, H.D. Rüter, E. Szilágyi, K. Temst, J. Magn. Magn. Mater., in press.

[28] L. Bottyán, J. Dekoster, L. Deák, B. Degroote, E. Kunnen, C. L’abbé, G. Langouche, O. Leupold, M. Major, J. Meersschaut, D.L. Nagy, R. Rüffer, in: ESRF Highlights 1999, European Synchrotron Radiation Facility, Grenoble 2000, p. 62.

[29] H.T. Hardner, M.B. Weissmann, S.S.P. Parkin, Appl. Phys. Lett. 67, 1938 (1995).

[30] M. Rührig, R. Schäfer, A. Hubert, R. Mosler, J.A. Wolf, S. Demokritov, P. Grünberg, Phys. Status Solidi A 125, 635 (1991).

[31] R. Schäfer, J. Magn. Magn. Mater. 148, 226 (1995).

[32] N. Persat, H.A.M. van den Berg, K. Cherifi-Khodjaoui, J. Appl. Phys. 81, 4748 (1997).

[33] S. Langridge, J. Schmalian, C.H. Marrows, D.T. Dekadjevi, B.J. Hickey, Phys. Rev. Lett. 85, 4964 (2000).

[34] G.P. Felcher, Physica B 192, 137 (1993).

[35] V. Lauter-Pasyuk, H.J. Lauter, B. Toperverg, O. Nikonov, E. Kravtsov, M.A. Milyaev, L. Romashev, V. Ustinov, Physica B 283, 194 (2000).

[36] T.P.A. Hase, I. Pape, B.K. Tanner, H. Dürr, E. Dudzik, G. van der Laan, C.H. Marrows, B.J. Hickey, Phys. Rev. B 61, R3792 (2000). 